\title{
Capacitive Gas-bubble Sensor for Solid Oxide Fuel Cell
}

\author{
A. Kobayashi ${ }^{* 1,2}$, H. Hiraoka ${ }^{1}$, and M. Yamaguchi ${ }^{2}$ \\ Ogihara Mfg. Co., Ltd., Japan, \\ *Corresponding author: Email kobayashi.a.og@gmail.com \\ ${ }^{2}$ Graduate School of Engineering, Iwate University, 4-3-5 Ueda, Morioka, 020-8551, Japan
}

\begin{abstract}
:
Solid oxide fuel cell (SOFC) is a type of hydrogen fuel cell for home use. A problem with SOFC is that carbon dioxide $\left(\mathrm{CO}_{2}\right)$ accompanies the hydrogen, when this is generated from natural gas by fuel reforming. In this study, we propose a gas-bubble sensor which is used in a battery-cell to prevent damage. The gas-bubble sensor has two detection electrodes wound around the outside of a cylindrical plastic housing. By measuring the changes in capacitance, the sensor can detect the presence or absence of gas-bubbles in the water. The water repellency of the plastic housing was improved chemically by using fluoroplastic (ethylene-tetrafluoroethylene copolymer; ETFE) so that the detection accuracy of the mixed gas-bubbles was maintained. As a result, a detection accuracy of $99 \%$ at an ultralow flow-rate of $50 \mu \mathrm{L} / \mathrm{s}$ was realized.
\end{abstract}

Key words: Solid oxide fuel cell, a capacitive gas-bubble sensor, fluoroplastic, water repellency

\section{Introduction}

The solid oxide fuel cell (SOFC) offers significant environmental benefits and high electrical efficiency [1, 2]. Unlike other fuel cells the SOFC is a solid-state device which operates at elevated temperatures. The principle of the electricity generation is the opposite of the electrolysis of water reaction, so that water is formed in the chemical reaction of hydrogen and oxygen for the generation of electricity.

A SOFC comprises a fuel reformer device to generate hydrogen by reaction of water and natural gas at high temperature.

The fuel reformer device generates hydrogen from natural gas by the following chemical equation:

$$
\begin{aligned}
& \mathrm{H}_{2} \mathrm{O}+\mathrm{CH}_{4} \rightarrow 3 \mathrm{H}_{2}+\mathrm{CO} \\
& \mathrm{H}_{2} \mathrm{O}+\mathrm{CO} \rightarrow \mathrm{H}_{2}+\mathrm{CO}_{2}
\end{aligned}
$$

Electrical power is generated from the hydrogen, and oxygen coming from the air as follows:

$\mathrm{O}^{2-}+\mathrm{H}_{2} \rightarrow \mathrm{H}_{2} \mathrm{O}+2 \mathrm{e}^{-}$

The SOFC has a water circulation system so that the processed water from the SOFC stack is reused in producing hydrogen (Fig.1). Thus the water is recycled efficiently. In addition to the hydrogen, there are many tiny carbon dioxide bubbles generated in the reaction, flowing in the pipes of the water circulation system. These tiny bubbles can combine and become a lager bubble. Large bubbles such as this can cause breakdown if they were to become bigger than $0.2 \mathrm{cc}$ and get trapped in the fuel reformer device, causing a blockage and preventing the water from flowing.

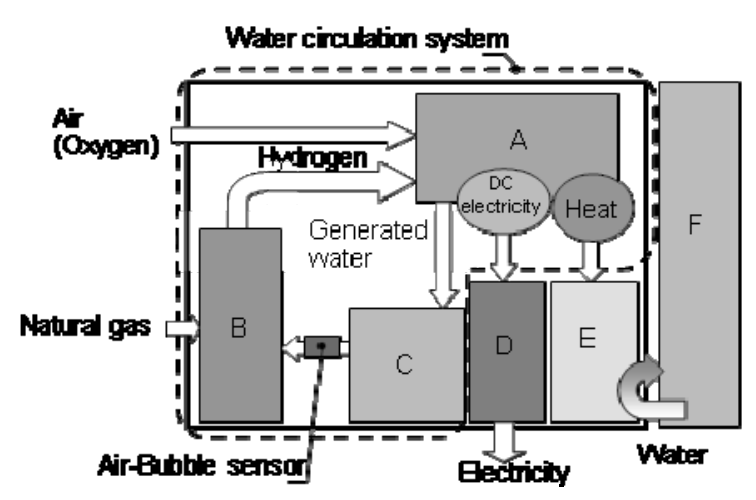

Fig. 1 Configuration of solid oxide fuel cell (A: SOFC stack, B: Fuel reformer device, C: Water treatment device, D: Inverter, E: Heat recovery system, F: Hot water storage tank).

In addition to this, because the SOFC system has a long start-stop time, like other power generation equipment, the system needs to be kept running at low level, even when generation of electricity is not needed. The system is always on, so that it can generate electricity efficiently when required. When the system works at a low level, the flow rate of the water circulation system decreases to $3 \mathrm{cc} / \mathrm{min}$., which is equivalent to a flow rate of $50 \mu \mathrm{L} / \mathrm{s}$. This is an ultra-low velocity flow. Therefore, it is 
necessary to detect the mixture of gas-bubbles and displacement of water with a probability of $99.9 \%$, at this ultra-low flow rate of $50 \mu \mathrm{L} / \mathrm{s}$.

In this study, a gas-bubble sensor, which is needed in order to develop the SOFC, is proposed. The sensor has to satisfy two requirements; i) impurities must not be eluted from the sensor device into the water, ii) it must have a high detection accuracy at the very low flow rate of $50 \mu \mathrm{L} / \mathrm{s}$. Fluoroplastic was used in order to chemically improve the water repellency of the housing material of the gasbubble sensor.

\section{Principle of the Gas-bubble sensor}

The gas-bubble sensor consists of a cylindrical plastic housing with an inner diameter of $4 \mathrm{~mm}$ and two electrodes wound on the outside of the housing. The area of each electrode is $150 \mathrm{~mm}^{2}$, and the distance between them is $13.5 \mathrm{~mm}$ (Fig.2A). The input voltage and frequency are $22 \mathrm{~V}$ and $9.7 \mathrm{kHz}$, respectively. An electric field occurs when a constant voltage is applied between the electrodes $A$ and $B$. The capacitance, or impedance, occurring between the electrodes is determined by the relative permittivity of water that fills the plastic housing. When air bubbles arrive in the electric field, the impedance is affected by the relative permittivity of air as well as the relative permittivity of water. The relative permittivity of air and water are 1 and 80.4 , so the impedance between the two electrodes changes with the presence or absence of air (Fig.2B)

\section{A. Configuration}

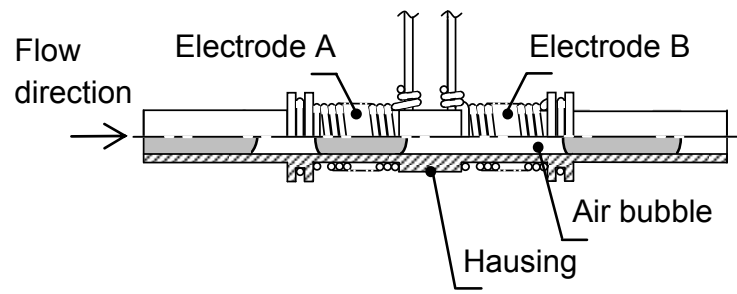

B. Measurement principle

( .......... : Line of electric force)

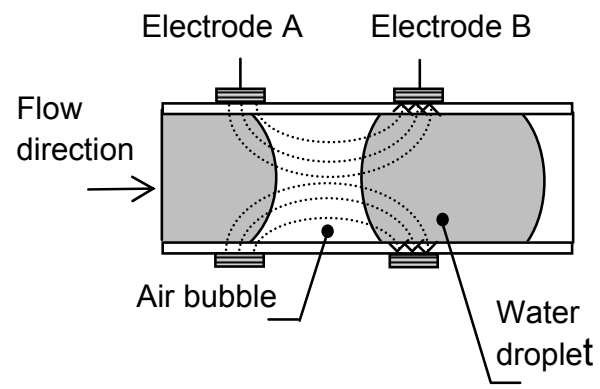

Fig. 2 Configuration of the capacity-type gas-bubble sensor and its measurement principle.
The detection circuit for the change in capacitance was shown in Fig.3. The current measured in the gas-bubble sensor is converted into a voltage by using a resistor.

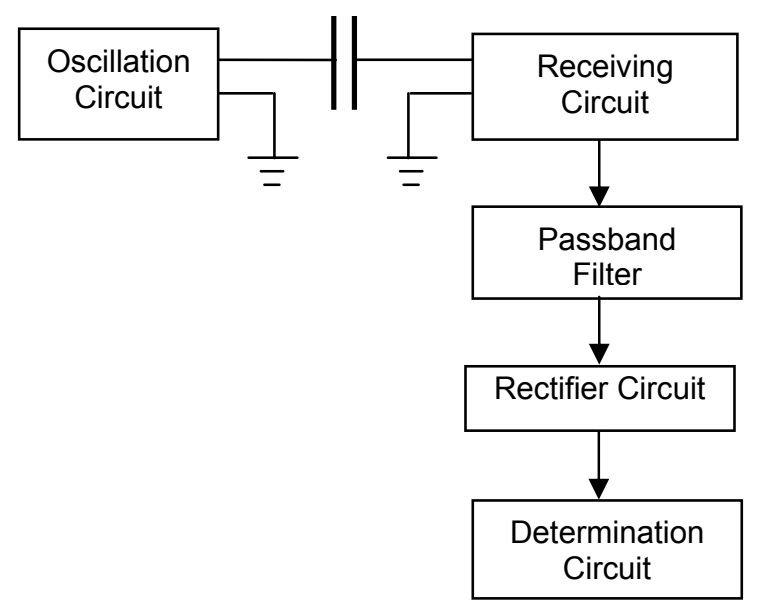

Fig.3 Circuit construction of the capacitive gasbubble sensor.

The alternating voltage is then rectified to a direct voltage using a full-wave rectifier circuit and a low pass filter. The setting the threshold value of the direct voltage is an important factor in determining the sensitivity of the sensor in detecting a specific size of air bubble. In this research, an output signal is obtained digitally and compared with a threshold level of $0.1 \mathrm{~V}$.

The relationship between the movements of gas-bubbles in a housing and detected voltage in consequence was shown in Fig.4. Water flows from electrode $A$ to electrode $B$ in the housing of gas-bubble sensor. The closer a gas-bubble approaches the electrode $B$, the lower the detected voltage becomes. The threshold value falls below $0.1 \mathrm{~V}$ and an output signal is produced when a gas-bubbles arrives mid-way between electrode $A$ and $B$. The detected voltage increases and the output signal stops as the gas-bubble moves away from the center of electrode $A$ and $B$.

\section{Water repellence of Gas-bubble Sensor}

However, it would increase the potential of false detection if gas-bubble became elongated in the housing. Therefore, it is necessary to use a chemical water repellent coating in the housing in order to maintain the round shape of the gas-bubble.

Also, it takes time to detect a gas-bubble when travelling through the housing untreated with water repellent; a gas-bubble spreads flat in the 


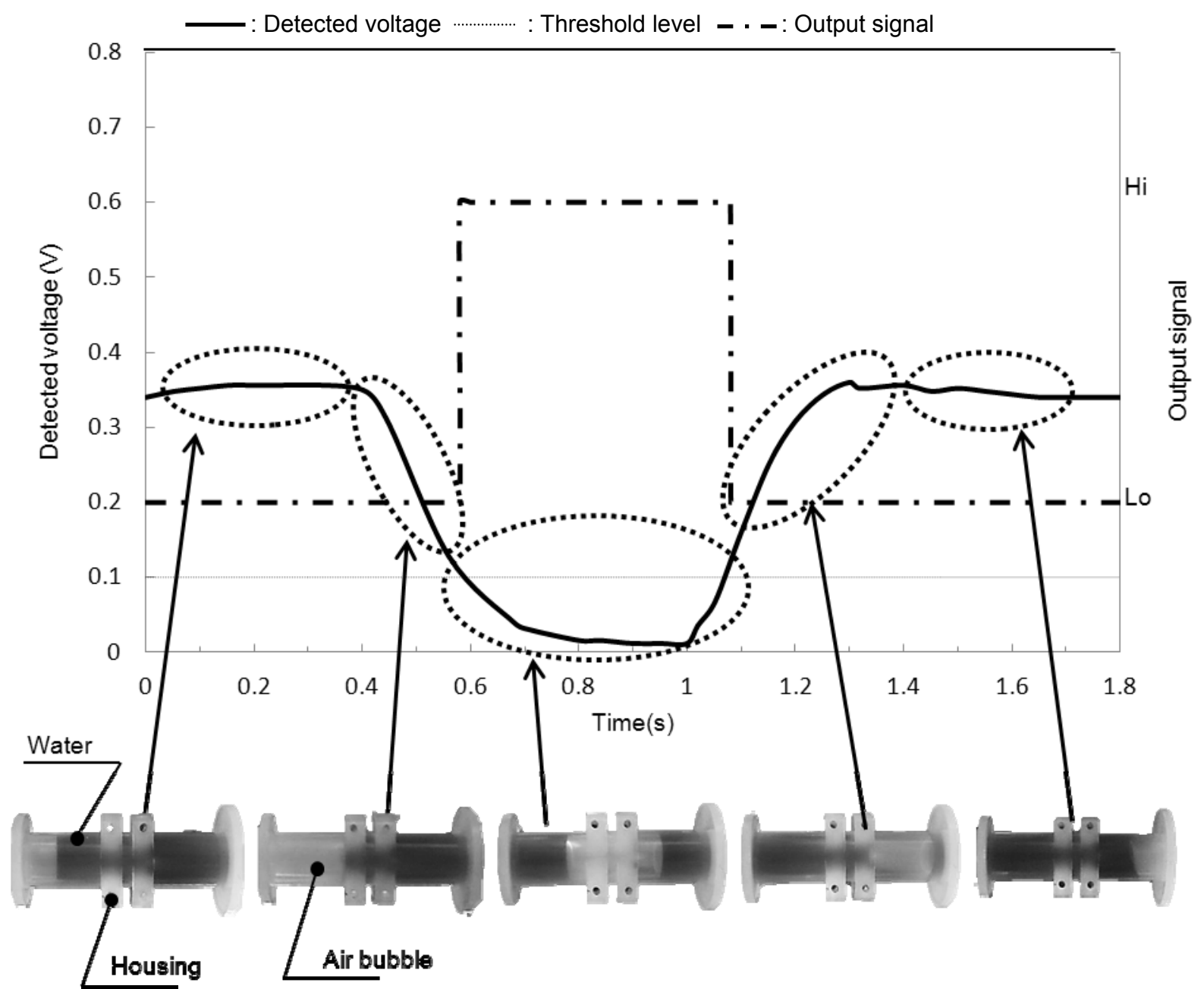

Fig. 4 Interrelationship between the movement of air in an ETFE housing and detected voltage .

housing, leaving a layer of water on the interior of the housing, which prevents a sufficient reduction in capacitance. On the other hand, the detection time becomes faster when the water repellence is high; water drops on the interior of housing are repelled when a gas-bubble and air pass by and the electrical capacitance is reduced.

Thus, the shape of the gas-bubble in the housing is a major factor in the accuracy with which the presence or absence of bubbles is detected. Therefore, the material of the plastic housing was considered in order to improve the chemical repellency of the water. The water repellent effect and the output signals at a flow rate of $50 \mu \mathrm{L} / \mathrm{s}$ were compared for two types of plastic housing, fluoroplastic (ETFE) and polyoxymethylene (POM).

In order to compare the water repellent characteristic of the two types of plastic housing, the static contact angle on the two materials was measured. To Measure the contact angles a digital microscope (VHX-1000, Keyence, 150x) and the open source image analysis software "Image J" were employed.

In order to evaluate the effect on the detection of the sensor due to differences in water repellency, compare the detection characteristics between ETFE and POM were compared. To aid measurement, $10 \mu \mathrm{S} / \mathrm{cm}$ purified water at a flow rate of $3 \mathrm{cc} / \mathrm{min}$. was introduced into a housing by a plunger pump, and inject approximately $0.2 \mathrm{cc}$ of air was injected by a syringe. The measured detection voltages and the output signals which occured when air passed through the detection area of the sensor were obtained with an digital oscilloscope (DP4054, Tektronix). 


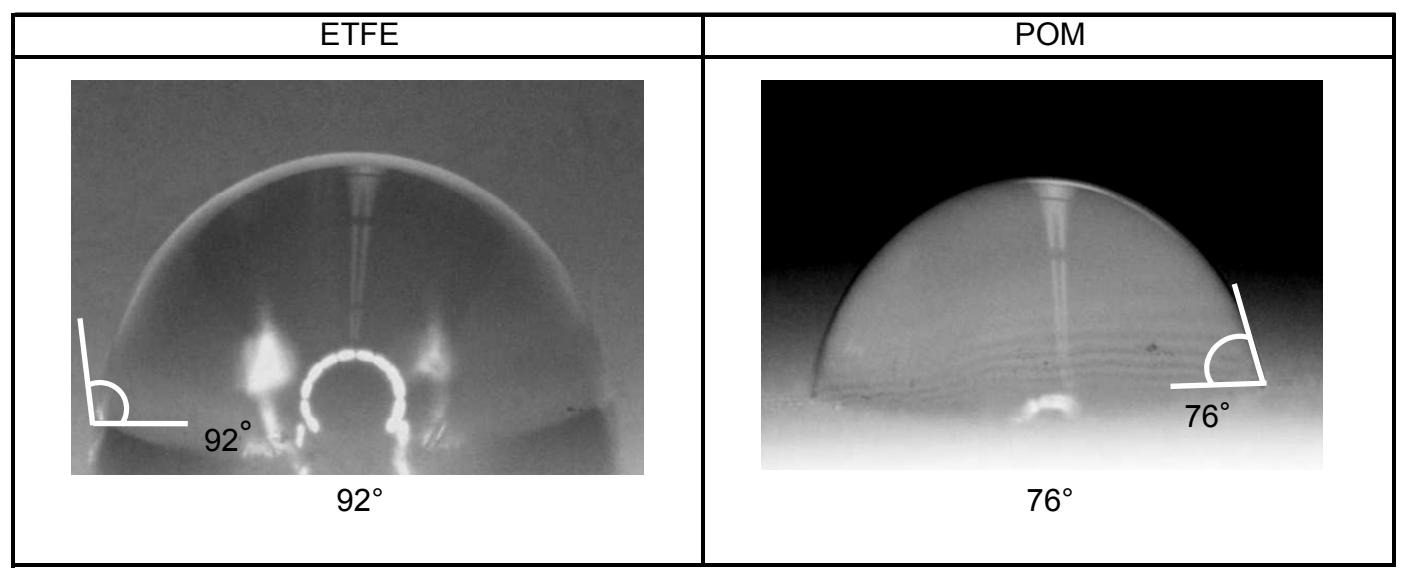

Fig. 5 Contact angles on POM housing and ETFE housing.

A. ETFE (contact angle: $92^{\circ}$ )

B. POM (contact angle: $76^{\circ}$ )

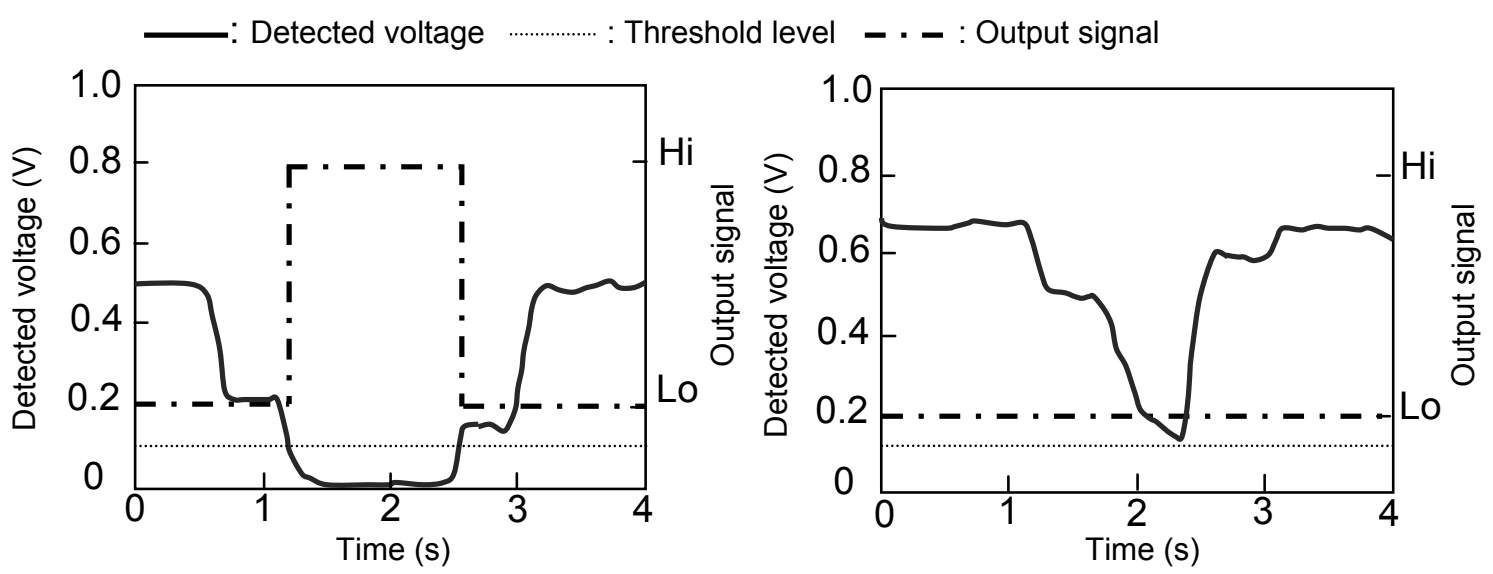

Fig. 6 Detected voltage and output signal characteristics of ETFE and POM.

\section{Results and Discussion}

The water contact angle of ETFE and POM were found to be $92^{\circ}$ and $76^{\circ}$ (Fig.5), respectively, showing that the ETFE is more water repellent. The calculated capacitances of ETFE and POM ranged between $0.31-7.12 \mathrm{pF}$ and $0.39-7.22 \mathrm{pF}$, respectively. Thus, little difference was observed between them. With ETFE, the detected voltages for water and gasbubbles were 0.508 and $0.012 \mathrm{~V}$, respectively, demonstrating that the gas-bubble sensor with the ETFE housing can detect gas-bubbles with an accuracy of $99.9 \%$ (Fig.6A). On the other hand, the sensor with the POM housing often failed to detect it (Fig.6B).

\section{Acknowledgement}

This research was supported by the Adaptable and Seamless Technology Transfer Program through target-driven R\&D, from Japan Science and Technology Agency, Japan.

\section{References}

[1] Ormerod RM, Solid oxide fuel cells, Chem Soc Rev 32(1), 17-28 (2003)

[2] Brett DJ, Kucernak AR, Aguiar P, Atkins SC, Brandon NP, Clague R, Cohen LF, Hinds G, Kalyvas C, Offer GJ, Ladewig B, Maher R, Marquis A, Shearing $P$, Vasileiadis $N$, Vesovic $\mathrm{V}$, What happens inside a fuel cell? Developing an experimental functional map of fuel cell performance, Chemphyschem 11(13), 2714-31 (2010) 